\title{
Revisión Bibliográfica y Extractos de Revistas
}

\section{LA PLACENTA Y LAS MEMBRANAS FETALES}

Por: Claude A. Villee y ol.

Editoral: Williams y Wilkins Company. Baltimore EE. UU. 1960.

Libro en edición de lujo de 400 páginas numerosas ilustraciones, la mayoría de las cuales son microfotografías.

Está dividido en cuatro partes y un índice. En la primera que comprende ocho capitulos se tratan puntos de gran importancia. La hormonología placentaria. Consideraciones histofísicas. Circulación placentaria. Función respiratoria placentaria. Función inmunológica.

Nutrición fetal, bioquimica y patológica.

La segunda parte, está constituida po: un resumen muy bien articulado de una conferencia en la que participan investigadores, obstétras, embriólogos y patólogos bien conocidos.

Finalmente se encuentran abundantes referencias biográficas sobre los temas desarrollados.

Este libro excelente, $\varsigma \mathrm{s}$ de importancia académica, y constituye, posiblemente, la más completa revisión que sobre placenta se haya hecho hasta el presente.

Son particularmente int resant:s las consideraciones sobre fi siología de la circulación placentaria y sobre la disposición vascular del órgano. 
E1 capítulo de Patología, aunque muy resumido, será de gran utilidad como fuente de información para los clínicos por lo c.aro y ordenado de su discusión.

Los estudios sobre Patología Placentaria en las enfermedades virales y la transmisión trasplacentaria de algunas viriasis, son también extraordinariamente interesantes y se encuentran ilustradas con excelentes fotomicrografias.

Este libro debe ocupar sitio destacado en la Biblioteca de los estudiosos de este campo tan superficialmente estudiado en la mayoría de los textos de Patología Obstétrica.

G. F.

\section{COMPLICACIONES DEL EMBARAZO}

\section{(médicas quirúrgicas y ginecológicas)}

\section{Por: El Equipo médico del Hospital Mount Sinai de N. Y. Drs. Alan G. Guttmacher, Joseph J. Rovinsky y Col. Editorial: Williams y Wilkins Company. Baltimore Estados Unidos. 1960.}

Libro en edición de lujo, que consta de seiscientas diecinueve páginas; dividido en catorce secciones o capitulos; en cuyo contenido se encuentran las siguientes materias: Sistema cardiovascular y renal, Sistema pulmonar. Sistema Gastrointestinal. Ojos, oidos, nariz y garganta. Problemas quirúrgicos. Problemas gin cológicos. Problemas Neurosiquiátricos. Problemas hematológicos. Disor denes endocrinos. Dermatología y sifilografia. Infecciones. Enfermedades del colágeno. Tumores malignos. Consideraciones genéticas.

Tan interesante obra, imprescindibl para todo especinlista en obstetricia, llena un gran vacío en la bibliografía consultable de la especialidad, pudiendo transcribir el valioso concepto que sobre él ha dado el Dr. Nicholson J. Eastman, Profesor de Obstetricia de la Universidad de Johns Hopkins de Baltimore: "Este volumen llena una gran necesidad. El embarazo se puede complicar con innumerables enfermedades intercurrentes, algunas comunes, otras raras. Qué influencia tendrá el embarazo sobre ellas? En qué forma influirán sobre el curso o evolución de él, y sobre el parto? Cómo deben ser tratadas? Este libro dará las respuestas más apropiadas a tales preguntas, analizando el curso de los procesos patológicos. Por tales motivos debo reconocerle un gran valor práctico para todo obstetra, así como también para el médico general abocado a tan variadas y problemáticas complicaciones". 


\section{MATERNIDAD Y MENSTRUACION SIN DOLOR}

\section{Educación de los hijos}

\section{Por: E. Dr. Gastavo Isaza Mejía. Medellín. Colombia.}

\section{Editorial: Bedout. Medellín, 1960.}

A manera de segunda cdición acaba de salir éste libro, en el cual además de los capítulos tratados en la primera, que apareció con el nombre de "La maternidad sin dolor", el autor creyó con veniente adicionar a más de la presentación de capítulos en forma de "clases", y en número de ocho (relativos al tratamiento de pacientes por el método sicoprofiláctico); dos partes más, una relativa a la aplicación de los principios pavlovianos al problema de las amenorreas, y otra por último, a un ensayo pedagóqico que bien puede tener cabida en un libro esencialmente popular, como el que comentamos.

Con las anteriores consideraciones, no dudamos en llamar la atención sobre la importancia de esta nueva contribución a la lucha por la higiene mental. Tal labor, emprendida hace ya largo tiempo por el destacado Profesor Dr. G. Isaza Mejía a través de sus publicaciones, está dedicado a prestar una ayuda más a todo obstctra que quiera servirse de ellos, para hacer más accesible a su cliente'a los principios y prácticas del método sicoprofiláctico.

M.A.F.B.

\section{TRANSFUSION DE SANGRE DE CADAVERES}

B. A. Petrov. Moscow, Surgery 46:4 Oct. 1959. Pag. 651

Se inicia el método con el experimento de Shanov en 1928 quien trasfundió perros con sangre de otros perros recién muertos. P stzriormente Yodin salvó $\mathrm{d}$ morir por hemorragia aguda a un suicida con 2 litros de sangre extraída a un cadáver de 6 horas.

En etapas sucesivas se vino a descubrir que la sangre de un muer- 
to no solamente permanece estéril por varias horas d spués de la muer te sino que permanece también funcionalmente efectiva como transportadora de oxígeno, función que persiste por 6 a 8 horas postm $\mathbf{r}$ tem. Los leucocitos retuvieron su acción fagocitócica por períodos hasta de 10 horas, ambos períodos de tiempo contados como previos a la extracción del cadáver.

Posteriormente se descubrió que la sangre de pacientes muer as de rcpente no se coagula o si lo hace es por corto tiempo, después 'el cual se hace líquida otra $v \in z$. Este fenómeno ofrece apreciab $1 \subset v \subset n-$ taja en la no necesidad de usar compuestos citratados para pr-servar la sangre almacenada.

Los cadáveres más aceptables como donantes son 'os de aq ellas personas que han muerto de repente pero no accidentalmente, pues en estos casos gran parte de la sangre extraída vendrá de los tejidos traumatizados con gran peligro de infección concomitante.

Técnica:

A) Traslado inmediato del cadáver al Hospital o Banco de Sangre.

B) Paciente en pssición extrema de Trend lemburg.

C) Disección de vena yugular izquierda y aplicación dz cánula dirigida hacia la cabeza.

D) Por gravedad la sangre fluy en recipientes estériles especiales en cantidad aproximada de 2 a 4 litros para un cadáver de unos 70 kilos.

Material: En. 28 años, el autor ha practicado más de 27.000 transfusiones de este tipo o lo que es lo mismo un $70 \%$ de todas las transfusiones en su hospital.

Ventajas: La autopsia practicada a los donantes antes de la transfusión al paciente, asegura a éste último la exclusión de t do peligro de trasmisión infecciosa alguna. Debido a la ausencia de citrato de sodio $\epsilon n$ las sangres trasfundidas, el porcentaje de reacciones a las mismas se reduce considerablemente.

J. V. M.

\title{
FUNCION TIROIDEA DURANTE EL EMBARAZO
}

\author{
William W. Engstrom. Marquette University Posgraduate Me- \\ dicine. Vol $27 \mathrm{~N}^{o} 2$ Feb. 1960.
}

Los autores resumen la función tiroidea durante el embarazo, $€ n$ la siguiente forma: 
1) Aumento de la capacidad de la Tiroxina para unirse a las protcínas sericas; 2) aumento de la concentración de la Yodoproteinemia; 3) disminución de la utilización de la tiroxina; 4) disminución breve del metabolismo basal; 5) aumento de la actividad tiroidea con un mayor aumento de la yodoproteinemia.

La tirotoxicosis se evidencia más comunmente en forma de la enfermedad de Graves y el diagnóstico se basa en el hallazgo de Met. Basal por encima de $30 \%$ de los valores normales y de una Yodoproteinemia por encima de $12 \%$.

El tratamiento de elección es mediante el uso de las drogas antitiroideas y la menos tóxica de ellas es Propiltiuracilo que debe adm:nistrarse a la dosis de $600 \mathrm{mgm}$ por dia en 4 dosis de $150 \mathrm{mgm}$. con las comidas y al acostarse.

Se debe ver a la paciente cada 4 semanas y no fiarse del criterio clínico o el metabolismo basal para el control del tratamiento, sino usar la Yodoproteincmia que se debe mantener entre $6 \sim 12 \%$.

Puesto que las drogas antitiroideas se eliminan por la leche, la paciente no debe amamantar su niño mientras esté recibiendo esta droga. Pasado el parto se debe contemplar tratamiento definitivo con cirugía o iodo radioactivo.

J. V. M.

\title{
HEMORRAGIA HABITUAL PREMENSTRUAL DESPUES DE ELECTROCAUTERIZACION CERVICAL
}

\author{
Louise Branscomb, M. D. Univ. de Alabama, Am. J. Obs. \& Gy-
} nec. Vol. 79 No 1 Enero 1960.

Observación del fenómeno ocasionalmente.

Material de estudio en 201 casos de hemorragia premenstrual: 11 d spués de electroconización y 190 después de electrocauterización cervical. 12 años.

$32 \%$ de las pacientes cauterizadas desarrollan la hemorragia premenstrual característica. Se presenta tanto en cauterizaciones endocervicales como ectocervicales. El intérvalo libre es la mayoría de los casos del 1 año. 
Sintomatología: comienza entre uno y siete días antes del comienzo de la menstruación y continua hasta el comienzo de la hemorragia, es de color carmelita sucio. Nunca se encuentra en otras épocas durante el mes o postcoito, no produce dolor, olor o irritación alguna.

Macroscópicamente: La lesión es más prominente inmediatamen te antes del comienzo de la hemorragia premenstrual. Se evidencia como una pequeña vesícula rojo oscura, ligeramente elevada. Estas vesículas se desarrollan sobre los puentes de mucosa dejados entre una y otra quemadura en el cuello o en el extremo de estas áreas de cauterización. Microscópicamente la lesión está cubierta de epitelio escamoso muy adelgazado. El estroma subyacente es edematoso.

Evolución: Generalmente una tercera parte de las pacientes dejan de sangrar después del primer año. El embarazo es curativo de la lesión. El tratamiento efectivo único es la conización del cuello con bisturí,

J. V. M.

\section{ACCION DE ALGUNOS NARCOBARBITURICOS SOBRE}

\section{LA MOTILIDAD UTERINA}

\section{G. Cerasuolo, G. Magli y S. Magiulo - Riv. D’Ostetricia e Ginecologia Practica. Nov, 1959 Paq. 1.023.}

Los autores ponen de presente la disparidad de criterios existentes en relación con la utilización clínica de los narcobarbitúricos en las intervenciones obstétricas y particularmente en la cesárea. A este respecto citan el trabajo de Calvanico acerca del uso de narcobarbitúricos en 250 cesáreas con resultados óptimos para la madre y el feto. En contraposición, mencionan la opinión de Mussey, Molinengo y Lauricella quienes anotan particulares cuidados en su empleo por los riesgos que implican.

Con el objeto de aclarar algunos puntos respecto a los efectos de los barbitúricos sobre la motilidad uterina, los autores han estudiado los efectos del Pentotal, Kemital, Baytinal, Citoeunarcon y Diogenal sobre la dinámica de los segmentos uterinos de ratonas al $18^{\circ}$ día de embarazo.

Para los distintos narcobarbitúricos estudiados y para concentraciones cercanas a las utilizables en clínica, se ha puesto en evidencia que el Baytinal carece casi completamente de acción sobre la actividad motora por cuanto en concentraciones de 1 x 30.303 escasa- 
mente eleva el tono, no modifica la frecuencia de las contracciones y apenas reduce en un $20 \%$ la amplitud de las mismas; mientras el Kemital y el Citoeunarcon intensifican el tono reduciendo la amplitud de las contracciones. El Pentotal y el Diogenal, por el contrario, disminuyen el tono, la amplitud y la frecuencia de las contracciones en el terreno experimental. Esto hace presumible que estos dos barbitúricos resulten perjudiciales en los casos de hipotonia e hipokinesia uterinas y en cambio puedan resultar útiles en las hipertonias e hiperkinesias,

J. A. G.

\title{
EXPERIENCIAS CON PEQUEÑAS DOSIS DE CORTISONA Y ESTEROIDES AFINES EN CASOS DE INFERTILIDAD ASOCIADA CON DISFUNCION OVARICA
}

\author{
Fertility and Sterility Vol. 11 № 11960. \\ William Mck. Jefferies M. D.
}

Reporta el autor el éxito obtenido en el tratamiento de 42 pacientes con infertilidad de origen ovárico disfuncional, mediante la ad. ministración prolongada de pequeñas dosis de Cortisona o Hidrocortisona.

El periodo de infertilidad previo al tratamiento, fué de 18 meses a 13 años. Las causas tumorales e inflamatorias de infertilidad se excluyeron previamente mediante exámenes apropiados. Todas las pacientes llevaron curvas de temperatura basal durante toda la duración del tratamiento.

E1 autor clasifica las 42 pacientes en cuatro (4) grupos, de acverdo con el grado de hirsutismo y el nivel de 17 Ketosteroides. La obtención de gestaciones fué la meta de este trabajo experimental.

Las dosis de Cortisona o Hidrocortisona variaron con cada paciente tratando en cada caso de mantener un nivel de excresión de 17 Ketosteroides, entre 8 y 10 miligramos por las 24 horas. En general, la mayoría de las enfermas respondieron exitosamente con dosis de $2.5 \mathrm{mgm}$ a $5 \mathrm{mgm}$, tres a cuatro veces al día.

En ninguna paciente se presentaron efectos indeseables con la administración prolongada de estas drogas.

Veintiocho (28) de las 42 pacientes previamente infertiles q edaron embarazadas durante el tratamiento. Los porcentajes fueron s.nsiblemente iguales ((??)) en los cuatro grupos. 
Debido a que $€ n$ buen número de las pacientes, los síntomas de disfunción ovarica se hicieron nuevamente presentes en períodos variables de tiempo luego de suspendida la administración de los corticoides, el autor sugiere el que algunas enfermas necesitarán terapéuticas practicamente indefinidas.

Concluye el autor diciendo que aunque falta por aprender acerca del mecanismo fisiológico de este tratamiento, él mismo ha probado ser un método efectivo y práctico para corregir casos previamente rebeldes de infertilidad.

J. V. M. 